orders of magnitude slower than commonly accepted average deposition rates of sediment in the deep sea ${ }^{2}$.

Scripps Institution of Oceanography, H. W. MENARD

University of California, La Jolla, California.

U.S. Navy Electronics Laboratory, C. J. SHIPEK San Diego, California.

'Murray, J., and Renard, A. F', “Deep-sea Deposits". H.M.S . Challenger, Sci. Results, 362 (1891).

${ }^{2}$ Goldberg, E. D., and Arrhenius, G. O. S., Geochim. et Cosmochim Acta, 13, 198 (1958).

\section{Diffusion of Zinc in Gallium Arsenide}

ZINC is an acceptor impurity in gallium arsenide with small ionization energy. If it is the predominant impurity and is present in low enough concentration, then the Fermi-level will lie above the zinc-levels and the zinc atoms will nearly all be ionized, that is, they will have accepted an electron. As the zinc concentration increases, the Fermi-level will descend into the valence band and the proportion of ionized atoms becomes small. Since the speed of diffusion of an atom will be different in its ionized and in its un-ionized state the diffusion coefficient will depend upon concentration.

The conditions under which one might observe this effect are as follows. We define a critical concentration $N_{\theta}$ of zinc atoms above which most of them will not be ionized, and below which most of them will be. Then we require the surface concentration to be greater than $N_{0}$ and the intrinsic number of holes and electrons to be small. These conditions can easily be satisfied for zinc diffusing into gallium arsenide at $1,000^{\circ} \mathrm{C}$. We have worked out the theory of this system and in Fig. 1 we show a theoretical curve of impurity concentration as a function of distance into a specimen when the diffusion is into a semi-infinite block with constant surface concentration of impurity. Also shown are some experimental points obtained by a radioactive tracer method. The agreement between theory and experiment is seen

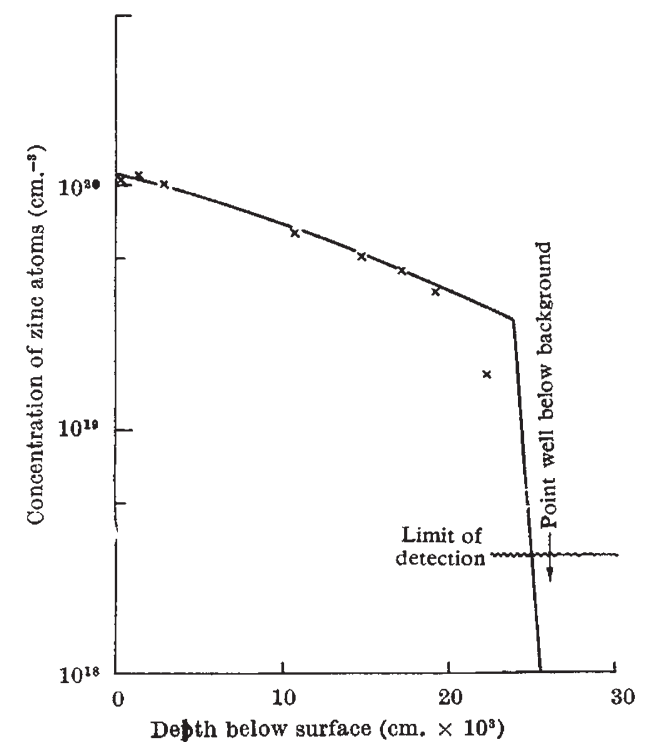

Fig. 1. Diffusion of zinc-65 in gallium arsenide. Temperature of diffusion $1,000^{\circ} \mathrm{C}$; ; time of diffusion, $3 \cdot 25 \mathrm{hr}$. curve. $\times$, Experimental points to be quite good. The most interesting feature of the curve is the change in slope when the concentration is equal to $N_{0}$, giving a very steep diffusion front, and this effect will obviously be of importance in design problems.

Two further points emerge which may be stressed. First, we can find from our analysis of the experimental results the diffusion coefficient of zine in both the ionized and the un-ionized states. Preliminary measurements show that at $1,000^{\circ} \mathrm{C}$. the diffusion coefficient of un-ionized zinc is about thirty times greater than that of ionized zinc. This is reasonable, since when the zinc has accepted an electron it is presumably more tightly bound and is also larger. We hope to find the two coefficients as a function of temperature in order to compare the activation energies. Secondly, the effects are particularly pronounced in the zinc-gallium arsenide system because the conditions enunciated above are well satisfied, but they may occur in other systems to a lesser degree and produce apparent anomalies. For example, it is quite possible to have an apparently normal concentration - depth curve which when extrapolated to the surface yields a value for the surface concentration considerably different from the true value. Full details of the theory and experiments and a discussion of these possibilities will be published elsewhere.

Acknowledgment is made to the Admiralty for permission to publish this communication.

J. W. ALLEN F. A. Cunnelt

Services Electronics Research Laboratory, Baldock, Herts. Aug. 25.

\section{Presence of 3 : 4-8 : 9-Dibenzpyrene in Coal- Tar}

Although several carcinogenic hydrocarbons of varied potency have been isolated from coal-tar, including $1: 2.5: 6$-dibenzanthracene, $3: 4$-benzpyrene $^{1}$, and $1: 12$-benzperylene ${ }^{2}$, it is known that the presence of such substances is not sufficient to account for both the qualitative and the quantitative carcinogenicity of coal-tar itself. This consideration warranted the search for further carcinogens in coaltar, particularly in pitch fractions containing the more condensed aromatic hydrocarbons, and especially in view of the considerable carcinogenic activity of several hexacyclic hydrocarbons such as $3: 4-8: 9$ $(\mathrm{I})^{3}$ and $3: 4-9: 10$-dibenzpyrene (II) ${ }^{4}$. Schoental ${ }^{5}$ has recently reported the presence of (II) in a carcinogenic fraction of coal-tar, and this prompted the present report of the isolation of another potent carcinogen, $3: 4-8: 9$-dibenzpyrene, from similar material. This hydrocarbon, which was isolated in small amounts by the usual fractionation and chromatographic methods, from the fractions boiling above $500^{\circ} \mathrm{C}$. of various samples of coal-tar pitch,

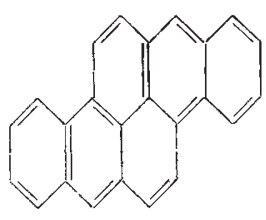

(1)

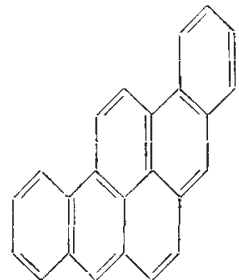

(II) 